\title{
Imaging Diagnosis and Treatment Progress of Metastatic Bone Tumor
}

\author{
Rui Jiang ${ }^{1}$, Ziyan Zhang ${ }^{2}$, Han $\mathrm{Wu}^{1}$, and Chao Zhang ${ }^{3}$ \\ ${ }^{1}$ Department of orthopedics, China-Japan Union Hospital of Jilin University, Jilin 130033, China \\ ${ }^{2}$ Department of orthopedics, The Second Hospital of Jilin University, Jilin 130041, China \\ ${ }^{3}$ Department of ophthalmology, The Second Hospital of Jilin University, Jilin 130041, China \\ Corresponding Author: Chao Zhang: zhangchao0701@163.com
}

Keywords: metastatic bone tumor; imaging examination; diagnosis and treatment; X-ray; CT; MRI and radionuclide imaging

\begin{abstract}
Metastatic bone tumors is a primary malignant tumor which is primary outside the bone. It belongs to a secondary malignant tumor spreading to the skeletal system through blood and lymphatic system. It is mostly discovered in elderly patients over the age of 40. The most metastatic tumors include prostate cancer, lung cancer, breast cancer, thyroid cancer, kidney cancer, etc. Presence or absence of bone metastatic tumor has very important sense to tumor staging, formulation of the best treatment plan, etc. Therefore, early detection as well as timely and effective treatment can obviously improve the patient's life quality of life, and increase the survival rate. Imaging examination is the most commonly used method for clinical diagnosis of bone metastatic tumor at present. The examination methods mainly include X-ray, CT, magnetic resonance imaging (MRI), radionuclide imaging, etc.
\end{abstract}

Any malignant tumor may be transferred to the skeleton system, and the adult metastatic bone tumor incidence is about 40 times of primary malignant bone tumor [1, 2]. Morphological diagnosis, including 'four fixed aspects' (accurate positioning, being qualitative, being quantitative locally and in diffusion scope as well as staging), provides comprehensive and valuable information about lesion in early stage. It is anatomy imaging foundation necessary for clinical operation and modern radiotherapy or chemotherapy, which is also related to acquisition of more effective treatment results and prognosis closely.

\section{The law between Malignant Tumor Diffusion and Bone Metastasis}

Anatomy and pathology prompt that bone metastasis probability is prominently increased with age. Autopsy confirms that the bone metastatic tumor incidence is up to 33 to $70 \%[1 \sim 4]$, the tumor is mostly multiple in dissemination through blood, and about $20 \%$ tumors can be single. There are no obvious symptoms in the early stage [2, 3]. Tumors prone to bone metastasis are clinically called pro0osseous tumor, such as prostate cancers (male patients account for about $60 \%$ metastasis), breast cancers (female patients account for about 70\%), lung cancer, thyroid cancer, nasopharynx cancer and kidney cancer, etc.; the bone invasion of myeloma and blood system tumor 
is also quite common. In addition to osteosarcoma, children and adolescents mostly suffer from neuroblastoma and blood system tumor osseous lesion. The proportion of cancer and sarcoma in bone metastasis is about 9:1 [1, 4].

Malignant tumor diffusion transfer is related to the decline of patient autoimmunity. It suggests that the oncocyte has higher portability to form genes or epigenetic mutation plasticity. It has more malignant (poorly differentiated) component for survival and development. It has stronger immune escape and angiogenesis activity for treating resistant cell clones. When the tumor is $3 \sim 4 \mathrm{~cm}$ long, new tumor blood vessels are required for blood supply. Once the blood vessel is formed, malignant tumors can achieve quick and chaotic proliferation in the tissue. Abnormal image features include the follows: mass effect, abnormal blood vessels in the focal area, surrounding invasion, edema, abnormal matrix and inflammation formation. It has been captured and confirmed by image dynamic enhancement scanning, more advanced sensitive function imaging, molecular specific contrast agent imaging, etc. [2, 4].

Cancer cell reaches bone tissue. Transforming growth factor - $\alpha$ (TGF- $\alpha$ ), $\beta$ and epidermal growth factor (EGF), etc. are released with bone cell growth. Osteoclast is activated with hyperplasia, and metabolism is strengthened prominently, thereby leading to bone destruction, oncocyte growth and pathological fracture. Oncocyte can also release osteoclast activator by stimulating tumor related immune cell, thereby causing bone destruction absorption. For example, malignant melanoma can produce activation factors such as TNF, IL- 1, GM- CGF, etc., thereby activating osteoclast. Medullary cavity bone trabecula erosion in bone marrow distribution area, osteolysis destruction and local bone density decrease appeared early. Therefore, invasion and propagation to surrounding area through Haversia pipes are available, the therapy also can induce bone mesenchyme, differentiate and generate into osteoblast [2, 5].

The manifestation types of bone neoplasm in different stages are diversified and complex, such as mall nodule, lump and large sheet. Some tumors undergo cystic changes [3, 4], which have a certain correlation with the complex pathological basis.

\section{The Image Method with Unique Value according to Different Principles.}

\subsection{X -ray inspection}

Plain film: it can display bone density change traditionally and basically. (1) Dissolving bony destruction is the main focus, which is mostly discovered in breast cancer, lung cancer, thyroid cancer, skin melanoma, etc. They are manifested as follows: wormy or patchy bony defect and bone density decrease. A few of tumors are osteoblastic, such as: prostate cancer accounting for about 90\%, lung cancer, adenoid cystic carcinoma, etc. The focus is mainly manifested as patches, nodules or cotton flocculation hyperplasia, and bone density is abnormally increased like gypsum. There is no periosteal reaction generally with less compression. The mixed type of osteolysis accompanied by osteogenesis is mostly discovered in breast cancer, which accounts for about 35\%.

(2) It involves cortex, which causes a variety of periosteal reactions. A few lung cancers are only accompanied with bone cortex damages in the early stage of metastasis. (3) Soft tissue mass is formed. (4) Collapse and pathological fracture are available, especially spine with dilatability compression.

Computed tomography (CT): more bone subtle change and surrounding soft tissue lesion information can be obtained compared with plain film and conventional CT. It is more valuable in basis craniil, chest wall, spine and pelvis for determining intramedullary and cortical range of focus, making clear the relationship between the tumor and the surrounding area, enhancing understanding on dynamic blood supply, and guiding puncture biopsy and surgical local treatment 
[6].

DSA and percutaneous coronary intervention: Tumor blood vessel imaging, including detected recurrent regenerated blood vessel, is used for identification of benign and malignant tumors. Direct percutaneous coronary intervention has particularly excellent effect in vascular bone tumor.

\subsection{MR Imaging}

Marrow and soft tissue dissection is displayed clearly with high resolution. The detection rate is higher than that of X-ray and bone scanning by $12.8 \%$. Because medullary cavity had rich blood pool and fat T1 and T2 weighted image (WI), they belong to high signals, which are well-controlled to bone destruction areas with relatively low signals. Early pathological changes have high sensitivity; STIR can inhibit normal high signal fat, and the lesion area (including edema zone) shows high or highlight signals. The positive rate is further improved [7]. In particular, the hematopoietic system lesion medullary cavity invasion, marrow replacement, substitution, etc. have certain characteristics. Multi-directional imaging shows spinal cord involvement in spinal canal directly. MRI is more likely to show contrast difference of soft tissue signal, which is beneficial to differential diagnosis. Blood vessels with flowing blood are directly displayed to enhance uneven strengthening like ;halo' at the edge of (Gd- DTPA) tumor focus.Totil Imaging Matrix (TIM) is available, parallel imaging (iPAT) is adopted for displaying systematic bone tissues in large scope at the same time. [10].

DWI has been applied in the body with the enhancement of high field strength and software technology. It is only most sensitive method to display physiological and pathological water molecule environmental changes in lesion cells. The unique edema in various cells during acute stage shows sensitive and highlight signal, and the ADC value can be measured in the corresponding apparent diffusion coefficient (ADC) diagram with certain quantitative reference value. The focus display is more sensitivve when background suppression Diffusion WIBS is inverted for shooting [9 14]. Spectral imaging also can be used for understanding the biological metabolic changes in bone destruction area.

MRI has no ionizing radiation.

\subsection{Radionuclide imaging}

Single photon emission compute tomography (SPECT) bone scanning. 99m Tc- MDP is mostly used as the tracer, and the technology is conventionally used for scanning and inspecting systematic bone lesions. 99m Tc- MDP participates in bone inorganic salt (hydroxyapatite) metabolism, which is absorbed by organic matters. Since tumors have invasive function metabolism changes, which appear earlier than anatomical morphology change by 3 to 6 months compared with ordinary X film. Focus concentration is related to osteoclast activity, the intake depends on the local osteotomy, Osteoclasts balance and body response after treatment [15].

Rradionuclide imaging is also used in tumor clinical treatment.

Positron Emission Computed Tomography (PET/CT): 18 F- FDG and 11 C - Methionine are commonly used as metabolism tracer. Ray imaging transmitted by positron emission nuclides entering into human body and participating in in-vivo biological activity is utilized. FDG has cellular transport capacity similar to glucose, which can be ingested by oncocytes. But it is not involved in further metabolism, which is stranded within the oncocyte. The commonly used semi-quantitative method includes standardized uptake value method (SUV), namely measurement of intake tracer concentration in the focus area, general quantitative concentration $<2.5$ is benign. 52 cases, including Aoki, confirmed that SUV can be used for effectively distinguishing between benign and malignant bone tumors, the average values of both groups are respectively 2.18 and 4.34 
(clinical results should be closely combined). The technology is cooperated with synchronous anastomosis CT thin layer computed tomography, PET and CT fusion image, thereby further improving the accuracy of 'four fixed aspects', lesion benign and malignant tumor identification, activities and inactivation degree provide important information. PET can be used for discovering more positive tumors in the relatively early stage of bone metastasis especially for individual bone destruction focus with unclear primary lesion and focuses with negative or suspected clinical manifestation in common examination [15 17].

The MAP diagram resolution of CT attenuation correction in PET/CT can reach 1 3mm. The sensitivity of PET/CT is higher than that of SPECT by 10 times. Currently, it is commonly recognized as one of the most sensitive imaging methods to eliminate late tumor metastasis. It is reported that the accuracy of lung cancer bone metastasis is more than 96\% [15 17]. Rosenbaum, Weder et al. reported that $14 \%$ bone metastasis can be detected after operation in stages, thereby significantly reducing the ineffective operation [15]. PET/CT is 'Discerned'.

\section{The Interconnection and Integration Relationship of New Imaging Methods and the Treatment Effect.}

\subsection{Multiplication of CT and PET fusion effect}

Multiplication of CT and PET fusion effect can directly reflect the biological metabolism of human body, PET capable of expressing biochemistry, physiological and pathological conditions in the body more accurately is combined with high-resolution CT. It is called 'greater power'. Israel, etc. compared the results of a group of late tumor cases before and after treatment. Respective unique values thereof are displayed: a total of 131 cases of malignant tumors were included, including 79 cases with bone metastasis, 296 bone focuses were discovered, CT and PET were compared, and the positive discovery was basically similar before treatment (though image performances are different). However, in the follow-up period after treatment: 282 focuses still can be discovered by CT, wherein most focuses showed ossification changes with density increase at different degrees. 172 focuses were displayed by PET. Though the quantity was less than that of CT in quantity, its function embodiment was better than that of CT. It was clinically confirmed that some focuses have been stable and inactivated, and PET indicated more satisfactory effect [17 18].

\subsection{Complementary role of ECT and PET}

The diagnostic accuracy of ECT bone scanning is about $66 \%$, which is related to image resolution and partial false positives $[15,16]$. In the early stage, bone reaction or smaller soluble bone lesion is not formed, it is too late to generate new bones due to high malignant tumor with too fast progress, such as malignant melanoma, etc. It can be expressed as false negative or 'cold zone'. Benign bone lesions, such as old fracture, bone hyperplasia etc., also can be manifested as abnormal concentration [16]. The uptake degree of FDG is related to the activity level of bone metastasis focus. 17 cases of prostate cancer bone metastasis of Morri-siv showed a total of 157 focuses. ECT and PET/CT were compared, a total of 95 focuses were discovered (71\%), including 31 focuses discovered by bone scanning merely (23\%), and 8 focuses discovered by PET/CT merely (6\%). However, the bone scanning showed that 30 cases were in a relatively stable state (30/31), PET/CT showed that focuses were in the active development period (8/8) according to follow-up. The latter was more significant obviously [19, 20]. The sensitivity of PET/CT and ECT bone scanning was about $65 \%$ and $90 \%$ respectively $[16,18]$. Clinical experience showed that 18F- FDG was often always sensitive to the osteolytic destruction. In addition, higher SUV value was also measured from the infection focuses, and clinical practice should be closely combined. 
Various tracers used for radionuclide imaging have their own specificity. 99m Tc-MDP is more sensitive to prostate cancer osteogenesis metastasis, osteosarcoma bone metastasis, etc. than 18 F-FDG [16, 17]. In addition, $11 \mathrm{C}$ - Met has high tumor/normal tissue ratio, it is especially more sensitive to brain tumors. The body inflammatory sties are not as concentrated as as sugar metabolites [17].

\subsection{The relationship between MRI dispersion weight and PET}

In diffusion weighted image (DWI), water molecule environmental change in tumor focus and acute edema area can show high signals, new malignant focus, malignant focus with rapid progress and surrounding abnormal signals are highlighted abnormally. The DWIBS shows 'high concentration' area similar to radionuclide imaging. Limited data reports MRI DWIBS and 18F FDG PET/CT. The display rate of the metastases focuses is similar in both technologies. The ADC value of MRI and the SUV value of PET have good correlation, namely $r=0.769, p<0.05$ in 65 metastases focuses [22]. MRI has no radiation injury, high sensitivity and no requirements on contrast agent. It has the disadvantage of limited specificity, no metabolic function information, less anatomical resolution than conventional scanning, serious interference with metal objects, etc. However, it is a meaningful and valuable exploration.

\subsection{Comparison of MR Angiography (MRA) and DSA}

MRA can show large and medium blood vessels naturally and non-invasively, including spinal and limb arteries and veins. Enhanced scanning is beneficial for improving small blood vessel display rate. It is convenient and safe during application in lesion primary screening, critical patient post-operative examination, etc. It is difficult to display microvessels in defects with paramagnetic interference. DSA is a minimally invasive method for angiography, which is the 'gold standard' for the diagnosis of vascular lesion. The tumor local microvascular changes are displayed selectively and clearly. Meanwhile, it also can play the role of treatment directly.

\section{Differential Diagnosis [23 26]}

\subsection{Identification of bone tumor benign and malignant tumors}

(1) Benign bone tumor is generally single with expansive growth and complete 'hardening edge'; enhanced scanning is not reinforced or uniformly reinforced; there is no soft tissue mass; edema belt is mild or unavailable, MRI dispersion weighted signal is not high, and the nuclide concentration is not obvious. (2) Malignant bone tumor invasive bone destruction and periosteal reaction are available. There is no 'hardening edge'. The enhanced scanning can be filled quickly, and the dynamic curve lifting slope is large with abnormal perfusion and uneven reinforcement. Obvious soft tissue mass is discovered; MRI dispersion weighted signal is high; the nuclide concentration is obvious. Pathological fracture line is irregular with dilatability compression and fast progress.

\subsection{Primary malignant tumor and metastatic identification}

(1) The primary malignant tumors are mostly composed of osteosarcoma, cartilage and fiber sarcoma. They are easily found before young and middle age. Cartilage and fiber sarcoma are also found in the early stage of old age. The tumors are mostly located on proximal end of long bone; MR T1WI and T2WI containing cartilaginous components have high signals. Chordomas and 
aneurysmal bone cysts are mostly located in the spine with obvious periosteal reaction, soft tissue mass and abnormal enhancement. (2) Metastatic tumor is generally multiple in the middle and old age. They are mostly discovered in vertebral arch root of longitudinal axis bone, attachment, pelvis, ribs and skull. The tumors in vertebra and flat bone are obviously more than that in long bone, which account for more than $90 \%$. Pathological fracture, periosteal reaction, soft tissue swelling and expansion as well as other changes are not as prominent as primary tumors. Single typical metastasis is not uncommon. Clinical practice should be closely combined for follow-up. Bone scanning metastasis focus has more obvious concentration.

\subsection{Bone multiple lesion identification}

In addition to the metastatic tumor, (1) leukemia, lymphoma, myeloma and other medullary cavity night lesions are considered malignantly, marrow replacement and substitution are formed in focal area, MRIT1WI normal high signal marrow is replaced by low signal tumor with own characteristics. The blood phase, serum immunoglobulin and Bence Jones protein abnormality are beneficial for determining the nature of myeloma. Pathological fracture, periosteal reaction (milder metastasis than primary focus), soft tissue swelling (primary malignant tumor, myeloma, skull rib bone metastasis and colon cancer systemic bone metastasis can be more obvious, and the rest is not obvious) and expansion (prostate cancer, kidney cancer, thyroid cancer and liver cancer are easily expanded) are relatively mild after bone severe damage. The abnormal concentration area is discovered in radionuclide bone. (2) Senile osteoporosis is mostly involved in centrum with non-swelling compression and no changes in soft tissues. If there is no fresh fracture, nuclide concentration and the enhancement of MRI STIR, and DWI signal are limited.

\subsection{Elimination of recurrence}

Recurrence is prompted by focal area expansion, edema aggravation, abnormal enhancement and discovery of abnormal new blood vessels. The recovery period is usually about $3 \sim 6$ months after the treatment of bone metastasis. PET, MRI dispersion weight and other examinations to embody functions and molecular level are advantageous in identification diagnosis and efficacy evaluation.

\section{Conclusion}

CT technology affects and leads rapid, comprehensive and scientific development stage of medical imaging, especially in-depth research on function and molecule level imaging; however, pathological diagnosis can not be replaced. Tumor focus as long as a few millimeters can be discovered and detected, the tumors may reach 'late stage'. The concept is constantly updated. Before the tumor is attacked, the doctor must fully understand and master the nature of the tumor focus, distribution of tumor in the patient body, activity and other detailed information firstly. Understanding of own and the enemy's situation should be achieved. Many imaging methods are increasingly showing joint, fusion and complementary trends. How to make the diagnosis correct and comprehensive should be jointly discussed, thereby implementing the tasks of more rational, accurate, timely and effective treatment. How to better apply limited equipment resources and funds in clinical purpose, and how to improve the diagnosis rate and curative effect still should be explored and summarized continuously.

\section{Acknowledgments}

This work was supported by the study of c-Myc and mTOR inhibitors in the combined targeting 
therapy of osteosarcoma. Jilin provincial finance department. (No 3D517P373430)

\section{References}

[1] Davies AM. Bone tumors,bone infection: radiologic- pathologic correlations. Eur Radio, 1999, Suppl 2:251,271

[2] Rubens RD,Bone metastases:the clinical problem. Eur J, Cancer, 1998, 34:210

[3] Eolelstyn GA,Gillespie PJ,Grebbel FS. The radiolocal demonstra-tion of osseous metastases: Experimental observations. Clin Radiol, 1967, 18: 158

[4] Matchell DG. Magnetic resonance imaging of musculoskeletal tu-mors: correlation with bone marrow biopsy. AJR, 1996, 166:137-140

[5] Edited by Jiang Hao. Bone and joint MRI. Shanghai: Shanghai Science and Technology Press, 1999.465-469

[6] Diederich S, Wormanns D,Semik M,et al. Screening for early lung cancer with low-dose spiral CT: Prevalence in 817 asymptomatic smokers.Radiology,2002,773

[7] Yang Kai, Wang Yali, Yang Jianping. Correlation between PIM-3 and p-STAT3 protein expression in osteosarcoma and benign bone tumor and clinical pathological significance. Chinese Journal of Practical Medicine, 2012, 39(14):73-75.

[8] Li Pin, Molecular pathologic study of bone tumor. Journal of Clinical Rational Drug Use, 2016,9(6):171-173.

[9] Yang Qingyan. Observation of application effect of X-ray in bone tumor diagnosis. China Health Standard Management, 2016, 7(11):176-178.

[10] Xu Hai. Study on the correlation between bone tumor imaging and pathology. Journal of Practical Cancer, 2016, 31(6):969-971.

[11] Qin Wen, Hu Yang. Application value of imaging examination in bone tumor diagnosis. Journal of Practical Cancer, 2016, 31(3):509-512.

[12] Fei Fei, Zhao Hai, Qu Lili. Overview of the latest interpretation of China bone tumor evidence-based clinical diagnosis and treatment guideline in 2015 on related new drug progress of malignant bone tumor, etc. Chinese Medical Science, 2016,6(2):5-6.

[13] Xie Fei. Comparison of application value of X-ray and CT in bone tumor patient diagnosis. Medical Information, 2015,28(20):324-325.

[14] Li Liangfeng. Exploration of the performance and clinical diagnosis of bone tumor in the radiographic plain film. World Latest Medical Information Digest, 2016,16(59):287.

[15] Wang Xifeng. Imaging diagnosis and progress of bone giant cell tumor. Journal of Clinical Medicine Literature,2016,3(23):4709-4710.

[16] Zhu Xiongzeng, Zhang Renyuan. Bone tumor pathological diagnosis basis. Journal of Clinical and Experimental Pathology, 1990,6(2):145-148.

[17] Amary MF,Bacsi K,Maggiani F,et al.IDH1 and IDH2 mutations are frequent events in central chondrosarcoma and central and periosteal chondromas but not in other mesenchymal tumours.J Pathol,2011,224(3):334-343.

[18] Chu Tongwei, Li Hong. Research progress of the molecular mechanism of metastatic bone tumor. China Bone Tumor Osteopathy, 2009,8(5):313-315.

[19] Walther C,Domanski HA,von Steyern FV,et al.Chromosome banding analysis of cells from fine-needle aspiration biopsy samples from soft tissue and bone tumors:is it clinically meaningful.Cancer Genet,2011,204(4):203-206.

[20] Ding Yi, Xi Yue, Sun Xiaoqi, et al. Comparison between stereo-tactic core needle biopsy and postoperative pathological diagnosis for bone cellule malignant tumors. Chinese Journal of Pathology, 2013, 42(3):163-166.

[21] Tanas MR,Sboner A,Oliveira AM,et al. Identification of a disease-defining gene fusion in epithelioid hemangioendothelioma.Sci Transl Med,2011,3(98):98.

[22]. Zhou Nina, Zhao Xuna, Chen Min. Comparative analysis and research on diagnosis of malignant tumor metastasis focus with DWIBS and PET. PHILIPS Clinical Viewer, 2007, 1:56.

[23] Lin Jinghui, Zhu Mei, Jiang Xuezhen, et al. Comparative study on bone metastasis focus detection with bone imaging and MRI. Chinese Journal of Nuclear Medicine, 2000, 20:184.

[24] Wang Jichen, Liu Shuxue, Wang Xiaoying. MRI and radionuclide analysis on prostate bone metastasis. Chinese Medical Imaging Technology, 2000, 16:191.

[25]. Mario Campanacci. Introduction bone and soft tissue tumors. New York: Springer-Verlag Wien, 1993.5- 31

[26]. Zhang Tianze, Xu Guangwei. Oncology. Tianjin: Tianjin Science and Technology Press, 2005.2162-VEGF Research on content correlation. Journal of Clinical Radiology, 2002, 21(1):38- 40 